\title{
Colecta e identificación de genotipos élite de granadilla (Passiflora ligularis Juss.) en Colombia
}

\section{Collect and identification of elite genotypes of sweet granadilla (Passiflora ligularis Juss.) in Colombia}
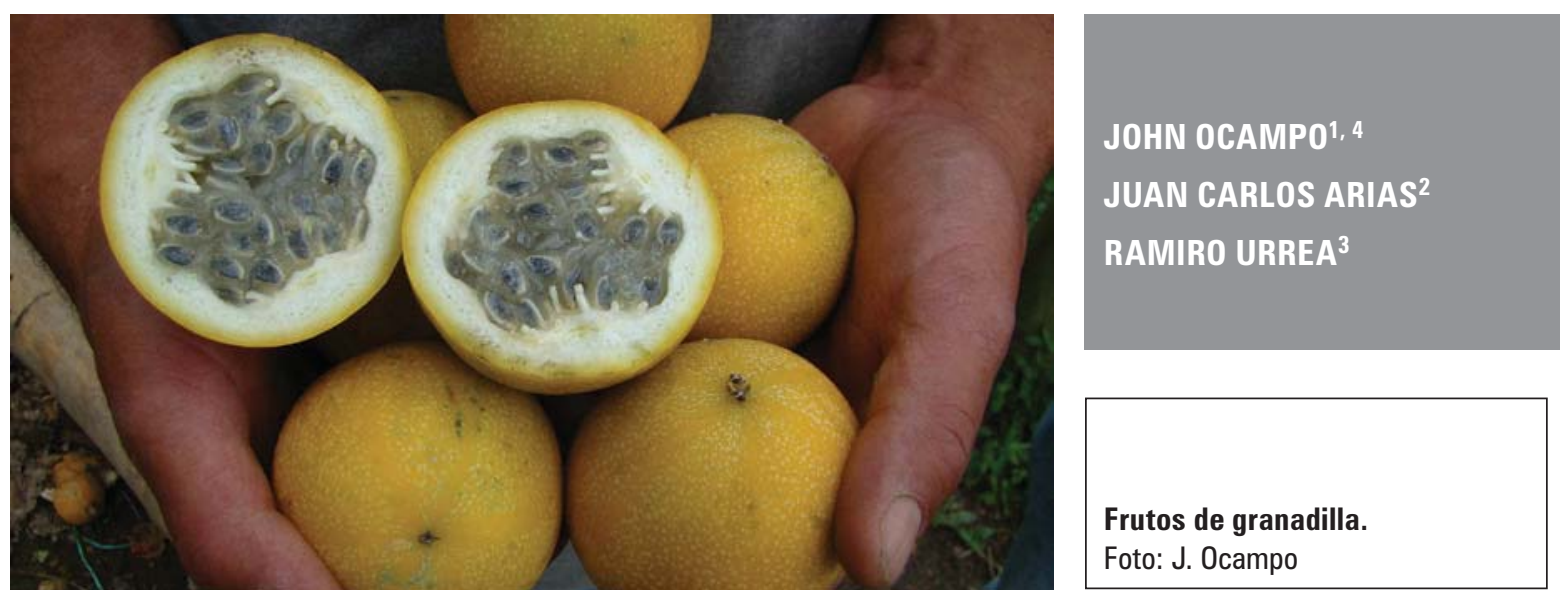

\section{RESUMEN}

La granadilla es la segunda especie en importancia económica del género Passiflora, por su fruto comestible y comercialización en los mercados internacionales. Sin embargo, existe poca información acerca de la variabilidad, lo que ha limitado un mejor desarrollo del cultivo. El objetivo del estudio fue colectar y caracterizar cultivos de granadilla para establecer el grado de variabilidad genética del fruto como base para el mejoramiento genético. Las colectas se realizaron en 35 municipios de 11 departamentos de la región andina entre 1.567 a $2.410 \mathrm{msnm}$ y en cada finca visitada se registró el manejo agronómico y el origen geográfico de la semilla. Diez frutos (calidad extra) por cultivo fueron seleccionados al azar de las plantas más sobresalientes (productividad y sanidad) con la participación de los productores. Análisis univariado y de clasificación (neighbor joining) fueron empleados en la caracterización del fruto con 11 variables fisicoquímicas. Los resultados identificaron a la mosca del ovario (Dasiops spp.) y antracnosis (Colletotrichum gloeosporioides) como los problemas fitosanitarios con mayor afectación en las 48 accesiones colectadas. La caracterización del fruto mostró un coeficiente de variación promedio total de 11,34\%, destacándose las variables peso de la cáscara $(14,61 \%)$ y el jugo (14,41\%). En cuanto a los parámetros de calidad, se identificaron siete accesiones élite con peso del fruto $>134 \mathrm{~g}$, ${ }^{\circ}$ Brix $>14,4$ y pulpa + semilla $>52 \%$. La clasificación arbórea estableció una relativa variabilidad intraespecífica con poca estructuración de origen geográfica entre las accesiones. Estas accesiones élite identificadas son la base para el inicio de un proceso de mejoramiento genético a partir de genotipos bien diferenciados y con características agronómicas de interés.

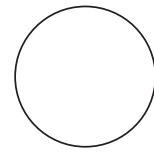

Palabras clave adicionales: Passifloraceae, recursos fitogenéticos, productores, fitomejoramiento.

Facultad de Ciencias Agropecuarias, Universidad Nacional de Colombia, Palmira / Centro Internacional de Agricultura Tropical (CIAT), Cali (Colombia).

2 Facultad de Ciencias Agropecuarias, Universidad Nacional de Colombia, Palmira (Colombia).

Facultad de Ciencias Agropecuarias, Universidad de Caldas, Manizales (Colombia).

4 Autor para correspondencia: jaocampop@unal.edu.co 


\section{ABSTRACT}

The sweet granadilla is the second-ranked species in the Passiflora genus in terms of economic importance due to its edibility and commercialization in international markets. However, there is little information on its variability, which has limited any betterment of crop development. The objective of this study was to collect and characterize passion fruit crops to establish the degree of genetic variability of this fruit for breeding purposes. Collections were made in 35 municipalities located in 11 departments of the Andean region, between 1,567 and $2,410 \mathrm{~m}$ a.s.l.; on each farm, the farm crop management and geographic origin of the seeds were registered. Ten fruits per crop were randomly selected (extra quality) from the outstanding plants (productivity and health) with the cooperation of the farmers. Univariate and classification (neighbor joining) analyses were used for the characterization of the fruits with 11 physicochemical variables. The results identified lonchaeid flies (Dasiops spp.) and anthracnose (Colletotrichum gloeosporioides) as the main phytosanitary problems in the 48 accessions collected. The fruit characterization showed a total average coefficient of variation of $11.34 \%$, with notable results for the variables of weight of the fruit peel $(14.61 \%)$ and the juice $(14.41 \%)$. For the quality parameters, seven elite accessions were identified, with fruit weight $>134 \mathrm{~g},{ }^{\circ}$ Brix $>14.4$ and pulp + seed $>52 \%$. The cluster analysis established a relative intraspecific variability with slight structuring due to geographic origin among the accessions. These elite accessions are the basis for beginning a breeding process with distinct genotypes and agronomic traits of interest.

Additional key words: Passifloraceae, plant genetic resource, farmers, plant breeding.

Fecha de recepción: 01-10-2014

Aprobado para publicación: 28-05-2015
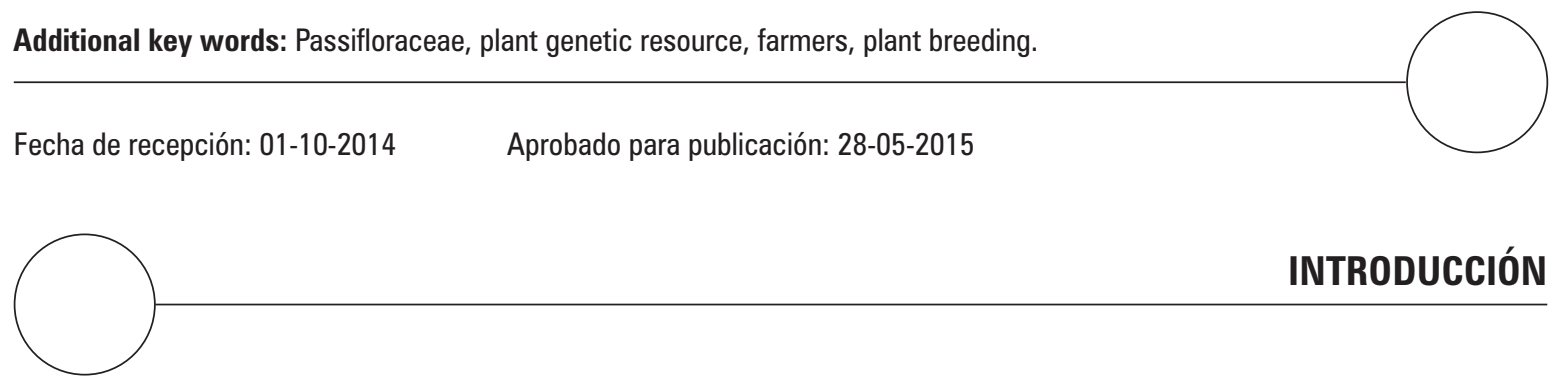

La granadilla (Passiflora ligularis Juss.) es la segunda especie de importancia económica del género Passiflora L. después del maracuyá (P. edulis $\mathrm{f}$. flavicarpa Degener), ya que los frutos son comercializados en mercados nacionales e internacionales por su agradable sabor y valor nutricional (Yockteng et al., 2011). La especie se distribuye desde México a Bolivia y principalmente en los Andes tropicales entre los 1.500 y $2.500 \mathrm{msnm}$ (Killip, 1938). Esta planta ha sido introducida a otros países como especie ornamental o frutal en Kenia y Nueva Zelanda. El número cromosómico de la granadilla es $2 n=18$ y un tamaño del genoma de 1,414 pg. (Snow y MacDougal, 1993; Yotoko et al., 2011). Las flores son hermafroditas y la polinización (alógama) es realizada principalmente por insectos de los géneros Xylocopa spp. y Epicharis spp. (Snow y MacDougal, 1993; Franco et al., 2007). Así mismo, la abundancia de los polinizadores en los cultivos de granadilla puede incrementar la productividad y la variabilidad de- bido al constante flujo genético por intercambio de polen entre plantas (Arias et al., 2015).

Los principales productores a nivel global son Colombia, Perú y Ecuador donde es cultivada por pequeños campesinos en zonas de ladera y cultivos no superiores a 1,5 ha en promedio ( $\mathrm{Pa}-$ rra, 2013). En Colombia la granadilla se cultiva hace más de 50 años, y los departamentos de Huila, Antioquia y Cundinamarca son los mayores productores con cerca de 3.700 ha (Agronet, 2014) y genera cerca de 210 jornales/ha-año (Parra et al., 2011). Esta fruta en los últimos años ha conquistado el mercado de algunos países europeos (Holanda, Bélgica, Francia, Portugal y Alemania), asiáticos (Emiratos Árabes y Hong Kong), norteamericanos (Canadá), centroamericanos (Costa Rica) y suramericanos (Brasil) contribuyendo considerablemente la economía campesina y con las exportaciones colombianas (Parra, 2013). 
A pesar de su importancia económica, las plantaciones se han visto afectadas por graves problemas fitosanitarios (Dasiops spp., Haematonectria haematococca, y virus SMV), degeneración genética, ausencia de viveros certificados y falta de mejoramiento genético en la especie (Ocampo y Wyckuys, 2010). Esto ha traído como consecuencia la reducción del ciclo del cultivo a 24 meses, aumento en los costos de producción y la disminución de los rendimientos, los cuales han pasado en los últimos años de 14 a $10 \mathrm{t} \mathrm{ha}^{-1}$ en promedio nacional (Parra et al., 2011; Agronet, 2014). En la actualidad, en la granadilla y el maracuyá los productores seleccionan los mejores frutos por medio de selección fenotípica de las plantas con mayor vigor para establecer los cultivos en función de sus observaciones de un fenotipo ideal para el mercado (Ocampo et al., 2013). Esta práctica puede contribuir con la disminución de la variabilidad en los cultivos por depresión endogámica en los siguientes ciclos de producción. Sin embargo, el síndrome de polinización cruzada, el intercambio de semillas entre productores y el amplio rango de distribución geográfica de los cultivos mantienen una considerable variabilidad en los genotipos cultivados en Colombia (Bernal et al., 2014).

Por otro lado, en la granadilla no se han reportado estudios de mejoramiento genético sistemático y la disponibilidad de un cultivar más rustico y productivo representaría un incremento significativo en los ingresos de los productores (Parra et al., 2011). Así mismo, el conocimiento de la variabilidad genética es fundamental para definir el valor potencial de los recursos genéticos y relación con los parientes silvestres, que permita enfrentar el riesgo a los problemas fitosanitarios (Hidalgo, 2003; Hammer et al., 2012).

En el género Passiflora L. se han realizado algunos estudios sobre la variabilidad a nivel intra e interespecífico en algunas especies cultivadas empleando marcadores fenotípicos (Medina et al., 2000; Ocampo et al., 2007) y del ADN (Segura et al., 2002; Ocampo et al., 2004, Santos et al., 2011), los cuales han establecido una alta variabilidad entre las especies. Sin embargo, el tamaño y constitución de la muestra han limitado la interpretación con mayor detalle de la variabilidad intraespecífica en la granadilla, respecto a sus especies relacionadas. En Colombia, Castañeda (2008) reporta el primer estudio de variabilidad genética en la granadilla empleando marcadores RAPD y AFLP con materiales cultivados en los departamentos de Caldas, Quindío y Risaralda. Estos resultados no revelan con claridad una estructura genética, debido a que los genotipos evaluados provienen de un mismo punto geográfico. En contraste, Bernal et al. (2014), empleando marcadores microsatélites con 41 accesiones colombianas, demostraron que la granadilla cultivada en Colombia posee un alto grado de variabilidad genética sin una estructura poblacional.

En términos generales, la poca estructura genética de la granadilla impide determinar las relaciones entre y dentro de accesiones de diferentes orígenes geográficos de acuerdo con los caracteres asociados al fruto, los cuales son los más relevantes para los procesos de selección de genotipos superiores. Por tal motivo, el objetivo de este estudio fue determinar el grado de variabilidad genética del fruto existente en la granadilla cultivada en Colombia proveniente de diferentes orígenes geográficos por medio de la colecta y la caracterización en fincas de productores para establecer las bases de un programa de fitomejoramiento.

\section{MATERIALES Y MÉTODOS}

\section{Área de estudio}

Las colectas de los materiales fueron realizadas entre los años 2009 y 2011 en 35 municipios de los departamentos de Antioquia, Boyacá, Caldas, Cauca, Cundinamarca, Huila, Putumayo, Quindío, Risaralda, Tolima y Valle del Cauca (tabla 1). La planificación de las recolecciones 
fue apoyada con las bases de datos de las Secretarías de Agricultura departamentales, la información de los productores y la distribución geográfica de las plantaciones de granadilla.

\section{Metodología de recolección}

En cada sitio de colecta se seleccionaron diez frutos de calidad extra de las plantas fenotípicamente más vigorosas con la participación de los productores de acuerdo con la longevidad $\geq 24$ meses, precocidad, producción, sanidad y calidad del fruto, empleando el método de selección masal por el fenotipo de acuerdo con Ocampo et al. (2013) y bajo los parámetros de calidad del Icontec (1997). Los frutos recolectados en cada finca constituyeron una accesión proveniente de polinización abierta constituida por genotipos de hermanos medios. Así mismo, en cada finca se registró el origen de la semilla, la edad del cultivo, el estado fitosanitario, la pendiente del terreno, el sistema de tutorado, el sistema de riego y la ubicación geográfica. La incidencia de insectos plaga y enfermedades se determinó tomando 12 plantas al azar en cada cultivo, los cuales fueron identificados a partir de la meto-

Tabla 1. Número de accesiones, departamentos y municipios donde se realizaron las colectas de germoplasma de granadilla en Colombia.

\begin{tabular}{|c|c|c|c|c|}
\hline No. & Código & Departamento & Municipio & $\begin{array}{l}\text { Altitud } \\
\text { (msnm) }\end{array}$ \\
\hline 1 & AntLig01 & Antioquia & Jardín & 2.029 \\
\hline 2 & AntLig02 & Antioquia & Urrao & 2.102 \\
\hline 3 & AntLig03 & Antioquia & Urrao & 1.864 \\
\hline 4 & BoyLig01 & Boyacá & Buenavista & 2.059 \\
\hline 5 & BoyLig02 & Boyacá & Buenavista & 1.972 \\
\hline 6 & BoyLig03 & Boyacá & Buenavista & 2.245 \\
\hline 7 & BoyLig04 & Boyacá & Tibaná & 2.164 \\
\hline 8 & BoyLig05 & Boyacá & Somondoco & 1.947 \\
\hline 9 & Callig01 & Caldas & Aranzazu & 2.144 \\
\hline 10 & CalLig02 & Caldas & Salamina & 2.084 \\
\hline 11 & CalLig03 & Caldas & Salamina & 2.212 \\
\hline 12 & Callig04 & Caldas & Aranzazu & 2.017 \\
\hline 13 & Callig05 & Caldas & Anserma & 1.883 \\
\hline 14 & Callig06 & Caldas & Anserma & 1.830 \\
\hline 15 & CauLig01 & Cauca & El Tambo & 1.812 \\
\hline 16 & CunLig01 & Cundinamarca & Venecia & 1.948 \\
\hline 17 & CunLig02 & Cundinamarca & Arbeláez & 1.990 \\
\hline 18 & CunLig03 & Cundinamarca & Fusagasuga & 1.890 \\
\hline 19 & CunLig04 & Cundinamarca & Pasca & 2.145 \\
\hline 20 & CunLig05 & Cundinamarca & Silvania & 2.201 \\
\hline 21 & CunLig06 & Cundinamarca & Choachi & 2.121 \\
\hline 22 & HuiLig01 & Huila & La Plata & 2.129 \\
\hline 23 & Huilig02 & Huila & La Plata & 2.091 \\
\hline 24 & Huilig03 & Huila & La Argentina & 2.009 \\
\hline
\end{tabular}

\begin{tabular}{|c|l|l|l|c|} 
No. & Código & Departamento & Municipio & $\begin{array}{c}\text { Altitud } \\
\text { (msnm) }\end{array}$ \\
\hline 25 & Huilig04 & Huila & Palestina & 1.948 \\
\hline 26 & HuiLig05 & Huila & Palestina & 1.770 \\
\hline 27 & Huilig06 & Huila & Pitalito & 1.892 \\
\hline 28 & Huilig07 & Huila & Garzón & 2.084 \\
\hline 29 & PutLig01 & Putumayo & Sibundoy & 2.111 \\
\hline 30 & Quilig01 & Quindío & Genova & 2.057 \\
\hline 31 & Quilig02 & Quindío & Genova & 1.887 \\
\hline 32 & Quilig03 & Quindío & Calarcá & 1.764 \\
\hline 33 & Quilig04 & Quindío & Circasia & 1.766 \\
\hline 34 & OuiLig05 & Quindío & Salento & 1.753 \\
\hline 35 & RisLig01 & Risaralda & Santa Rosa & 1.970 \\
\hline 36 & RisLig02 & Risaralda & Santa Rosa & 2.043 \\
\hline 37 & RisLig03 & Risaralda & Apia & 2.046 \\
\hline 38 & RisLig04 & Risaralda & Apia & 1.923 \\
\hline 39 & TolLig01 & Tolima & Cajamarca & 2.410 \\
\hline 40 & TolLig02 & Tolima & Cajamarca & 2.171 \\
\hline 41 & TolLig03 & Tolima & Ibagué & 1.885 \\
\hline 42 & ValLig01 & Valle del Cauca & Yotoco & 1.567 \\
\hline 43 & ValLig02 & Valle del Cauca & Restrepo & 1.573 \\
\hline 44 & ValLig03 & Valle del Cauca & Tulua & 1.967 \\
\hline 45 & ValLig04 & Valle del Cauca & Ginebra & 1.988 \\
\hline 46 & ValLig05 & Valle del Cauca & Versalles & 1.823 \\
\hline 47 & ValLig06 & Valle del Cauca & Versalles & 1.864 \\
\hline 48 & ValLig07 & Valle del Cauca & El Cerrito & 2.255 \\
\hline
\end{tabular}


dología propuesta por Fischer y Rezende (2008) y guías de campo (Tamayo y Varón, 1996; Rivera et al., 2002). Adicionalmente, se tomaron muestras de tejido vegetal afectado e individuos de insectos plagas para ser determinados en los laboratorios de fitopatología y entomología de la Universidad de Caldas y el Centro Internacional de Agricultura Tropical (CIAT) cuando no fue posible identificarlas.

\section{Análisis de datos}

Los frutos colectados fueron caracterizados con la medición de once variables fisicoquímicas cuantitativas (tabla 2) y los datos fueron analizados mediante la descomposición de la varianza (univariada), correlación de Pearson ( $r$ ) y el análisis de clasificación del "vecino más próximo" (neighbour joining) mediante distancias euclidianas. Los promedios de las variables fueron analizados mediante la prueba de comparación de Duncan (HSD) a un nivel de significancia del 5\%. Los análisis fueron desarrollados con los programas estadísticos Statistica v.8 (Hill y Lewicki, 2007) y Darwin v.6. (Perrier y Jacquemoud-Collet, 2006).

Tabla 2. Variables cuantitativas en la caracterización fisicoquímica de los frutos de granadilla.

\begin{tabular}{|c|l|c|}
\hline No. & \multicolumn{1}{|c|}{ Descriptor } & Código \\
\hline 1 & Peso del fruto (g) & PFR \\
\hline 2 & Longitud del fruto (mm) & LFR \\
\hline 3 & Diámetro del fruto (mm) & DFR \\
\hline 4 & Peso de la cáscara (g) & PCS \\
\hline 5 & Peso de la pulpa + semilla (g) & PPS \\
\hline 6 & Porcentaje de pulpa (\%) & \%PS \\
\hline 7 & Peso del jugo (g) & PJU \\
\hline 8 & Peso de la semilla (g) & PSEM \\
\hline 9 & Semillas por fruto & SEF \\
\hline 10 & Índice de semilla (g/100 semillas) & IS \\
\hline 11 & Sólidos solubles totales (1 a 20) & SST ('Brix) \\
\hline
\end{tabular}

\section{RESULTADOS}

\section{Características de los cultivos}

Un total de 61 fincas fueron visitadas entre los 1.567 y 2.410 msnm, de las cuales se seleccionaron 48 en 35 municipios de 11 departamentos que cumplían con los requisitos de sanidad, precocidad, rendimiento y edad de cultivo (figura 1). El mayor número de plantaciones se registró entre los 1.740 a 2.240 msnm (92\%) en zonas con pendientes superiores al $60 \%$ y bajo el sistema de tutorado en emparrado en la mayoría de los cultivos (96\%). La madera más utilizada como postes en el tutorado fue la guadua (Guadua angustifolia Kunth) y algunos productores en el departamento del Huila emplean el roble (Quercus humboldtii Bonpl), lo que ha conllevado a la deforestación intensiva de esta especie. La mayoría de los productores prefieren densidades de siembra por debajo de las 500 plantas/ha (80\%), siendo la máxima de 800 y la mínima de 156 plantas/ha cuando están asociados con el cultivo del café (Coffea arabica L.) en las primeras etapas de desarrollo. En las fincas visitadas no fueron registrados sistemas de riego y los cultivos dependen de la oferta hídrica de la precipitación. En cuanto al origen de la semilla, fueron identificados cuatro centros principales de dispersión con la información suministrada por los productores en cuatro departamentos, Urrao (Antioquia), Génova (Quindío), Buenavista (Boyacá) y Palestina (Huila).

En las localidades visitadas se registraron diferentes problemas fitosanitarios afectando los cultivos (figura 2). Se hallaron seis insectos plaga asociados con mosca del botón floral (Dasiops spp.), trips (Thrips sp.), ácaros (Tetranychus urticae Koch), chinche (Leptoglosus sp.), mosca negra (Drosophila spp.) y barrenador del tallo (Aepitus serta Schaus). El mismo número de enfermedades fueron registradas: ojo de pollo (Phomopsis sp.), virus de la hoja morada (Soybean Mosaic Virus - SMV), alternaria (Alternaria passiflorae J.H. Simmonds), secadera [Haematonectria haematococca (Berk. \& Broome) Samuels \& Rossman, anamorfo: Fusarium solani 


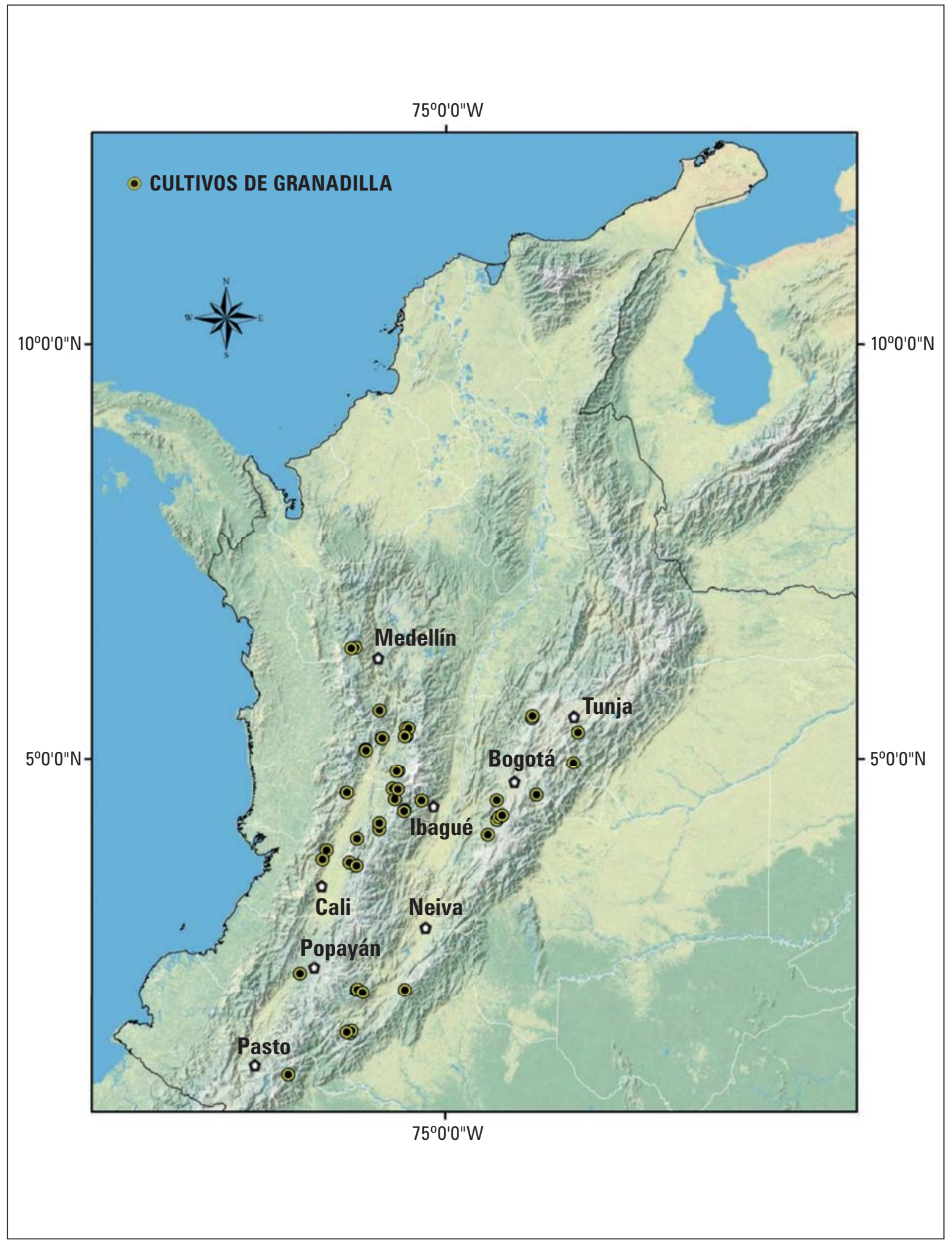

Figura 1. Distribución geográfica de las 48 plantaciones de granadilla (P. ligularis) seleccionadas para el estudio. 


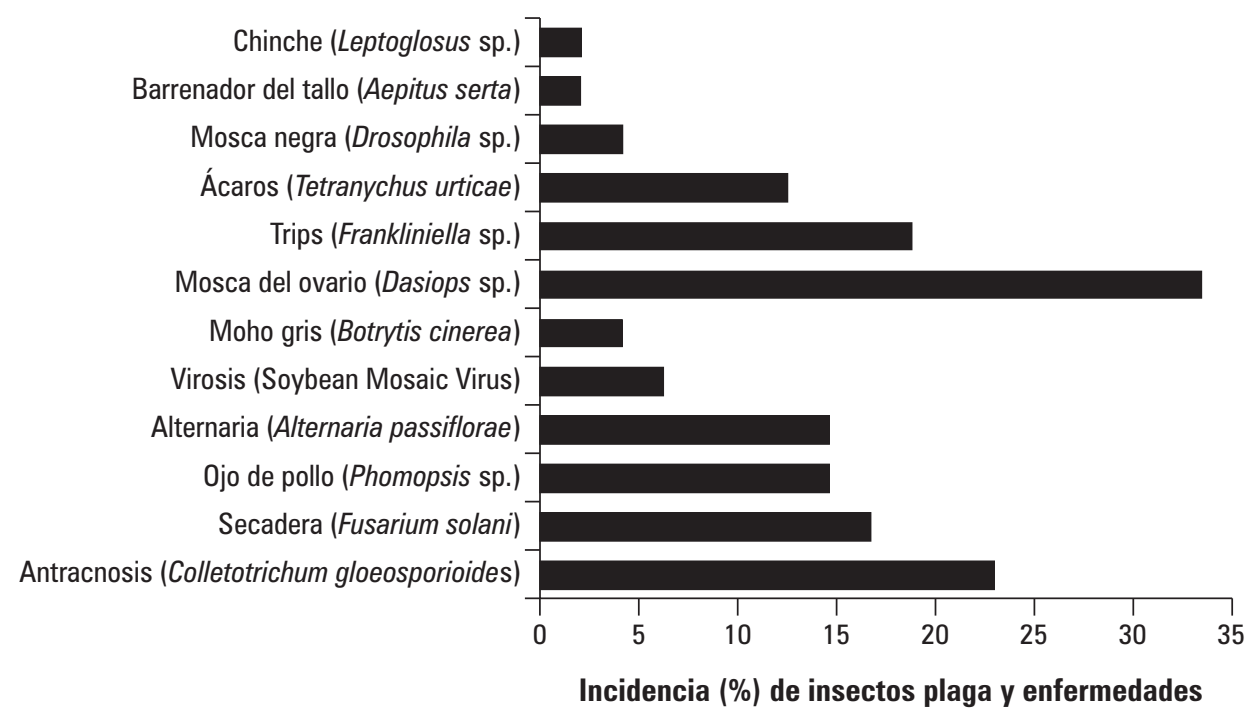

Figura 2. Principales plagas y enfermedades presentes en las muestras de cultivos de granadilla.

Mart. Sacc.], moho gris (Botrytis cinerea Pers ex Fr.) y antracnosis (Glomerella cingulata Stoneman, anamorfo: Colletotrichum gloeosporioides Penz). En el caso de los insectos plaga, la mosca del botón floral fue la de mayor importancia con una incidencia del $34 \%$, seguida por los trips con un 19\%. De igual manera, antracnosis fue la más observada con un $23 \%$ de incidencia y en especial en las zonas de mayor humedad relativa. Así mismo, secadera, ojo de pollo y alternaria fueron otros patógenos que tuvieron incidencias similares variando entre 14 y $17 \%$ en las fincas visitadas, sin que exista en ellas una preferencia por un piso térmico o región específica.

\section{Análisis fisicoquímico del fruto}

El análisis univariado mostró un coeficiente de variación (CV) promedio de 11,34\% para las 11 variables evaluadas, lo que indica una relativa variación fenotípica del fruto de las accesiones recolectadas (tabla 3). Las variables que más explican la variabilidad total están asociadas principalmente con los pesos de la cáscara (PCS

Tabla 3. Análisis univariado de 11 variables fisicoquímicas evaluadas en el fruto de granadilla.

\begin{tabular}{|c|c|c|c|c|c|c|c|c|c|c|c|}
\hline \multirow[b]{2}{*}{ Parámetro } & \multicolumn{11}{|c|}{ Variables $^{1}$} \\
\hline & $\begin{array}{l}\text { PFR } \\
\text { (g) }\end{array}$ & $\begin{array}{l}\text { LFR } \\
(\mathrm{mm})\end{array}$ & $\begin{array}{c}\text { DFR } \\
(\mathrm{mm})\end{array}$ & $\begin{array}{l}\text { PCAS } \\
\text { (g) }\end{array}$ & $\begin{array}{l}\text { PPS } \\
\text { (g) }\end{array}$ & $\begin{array}{l}\text { PPS } \\
(\%)\end{array}$ & $\begin{array}{l}\text { PJU } \\
\text { (g) }\end{array}$ & $\begin{array}{l}\text { PSEM } \\
\text { (g) }\end{array}$ & $\begin{array}{l}\text { SEF } \\
\text { (g) }\end{array}$ & $\begin{array}{c}\text { IS } \\
(100 \mathrm{~s})\end{array}$ & $\begin{array}{c}\text { SST } \\
\left({ }^{\circ} \text { Brix }\right)\end{array}$ \\
\hline Media & 142,65 & 80,56 & 72,27 & 70,01 & 72,64 & 51,23 & 63,59 & 9,04 & 304,64 & 2,98 & 13,73 \\
\hline Mínimo & 83,60 & 71,20 & 58,80 & 29,00 & 44,00 & 40,28 & 37,20 & 4,80 & 172,66 & 2,27 & 10,00 \\
\hline Máximo & 178,40 & 91,20 & 80,60 & 97,80 & 90,80 & 61,25 & 80,40 & 11,20 & 436,43 & 4,27 & 17,90 \\
\hline Desv. Est. & 17,18 & 4,25 & 4,44 & 10,23 & 9,85 & 4,61 & 9,16 & 1,22 & 42,06 & 0,34 & 1,54 \\
\hline Coef. Var. (\%) & 12,05 & 5,28 & 6,14 & 14,61 & 13,56 & 9,00 & 14,41 & 13,45 & 13,81 & 11,26 & 11,22 \\
\hline
\end{tabular}

${ }^{1} \mathrm{E}$ significado de las variables aparece en la tabla 2. 
14,61\%) y del jugo (PJU 14,41\%). De igual manera, se observó una escasa variación en los caracteres relacionados a las dimensiones del fruto (longitud y diámetro), la cual no superó el 7\%. Las variables con mayor coeficiente de variación (PCS, PJU) se utilizaron en la identificación y selección de plantas con características superiores y de esta manera alcanzar un mayor progreso genético por ciclo de selección.

En adición, el análisis de correlación $(r)$ realizado mostró una alta asociación del peso del fruto (PFR) con las demás variables $(r \geq 0,63)$, a excepción del contenido de ${ }^{\circ}$ Brix, semillas por fruto (SEF), índice de semilla (IS) y porcentaje de pulpa+semilla (\%PPS). Las claras asociaciones fenotípicas entre las variables de calidad del fruto (peso, tamaño, contenido de pulpa) sirven como elementos importantes para la selección dirigida de materiales sobresalientes o élites. Sin embargo, las bajas correlaciones $(r \leq 0,35)$, de la variable "sólidos solubles totales" ( ${ }^{\circ}$ Brix) con las de calidad sugieren que este parámetro tiene gran influencia con la interacción genotipo $x$ medio ambiente donde los cultivos están establecidos (Flórez et al., 2012).
Los parámetros de calidad, porcentaje de pulpa+semilla $(>50 \%)$, porcentaje de jugo $(>50 \%)$, peso del fruto $(>150 \mathrm{~g}) \mathrm{y}{ }^{\circ}$ Brix $(>14,5)$ permitieron identificar siete accesiones élite (figura 3). Estas fueron colectadas en los departamentos del Valle del Cauca (ValLig04), Cundinamarca (CunLig02, CunLig03 y CunLig06), Putumayo (PutLig01), Quindío (QuiLig04) y Tolima (TolLig03). Respecto a la prueba de comparación entre promedios de Duncan ( $\mathrm{P} \leq 0$, $05)$, las accesiones muestran diferenciación dentro de la mayoría de las variables a excepción de \%pulpa+semilla, la cual no mostró diferencias. La variable peso del fruto (PFR) presentó la mayor diferenciación entre las accesiones y solamente CunLig03 y 06 se asociaron sin desigualdad. Los sólidos solubles totales ( ${ }^{\circ} \mathrm{Brix}$ ) es una parámetro con influencia ambiental y, a pesar de que las accesiones provienen de diferentes orígenes, mantienen cierta igualdad. Aunque solamente el $13 \%$ de las accesiones colectadas mostraron calidad del fruto, su amplia distribución geográfica y las condiciones ecoclimáticas específicas de cada región, sugiere una relativa plasticidad genética y fenotípica de los materiales cultivados en Colombia.

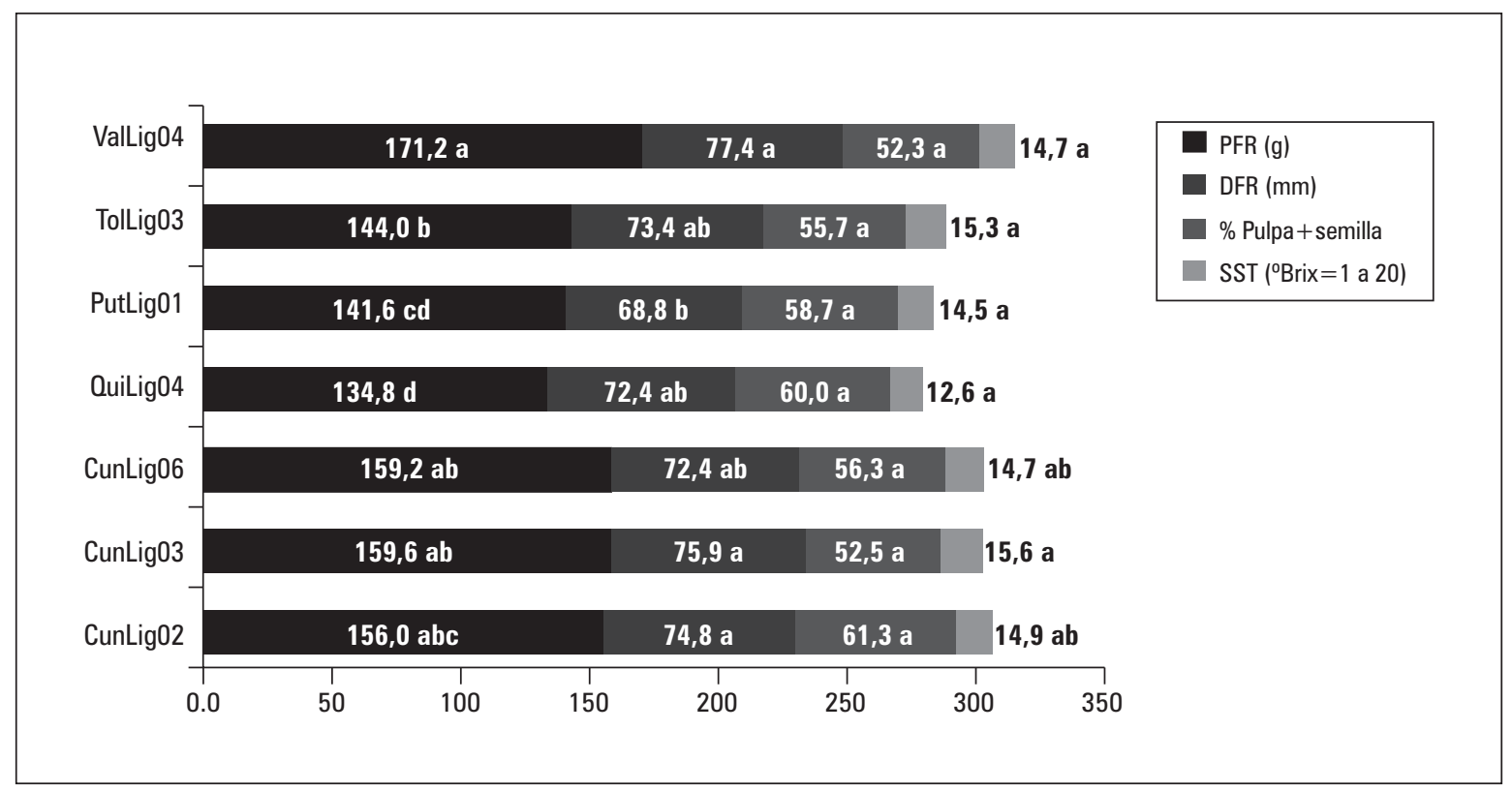

Figura 3. Características de calidad del fruto de siete accesiones élite de granadilla. Promedios con letras distintas indican diferencia significativa según la prueba de Duncan $(P \leq 0,05)$. 


\section{Análisis de la variabilidad}

El análisis de clasificación arbórea mostró cuatro ramas principales con alta heterogeneidad entre $\mathrm{y}$ dentro los individuos de las accesiones (figura 4). Esta variabilidad observada no muestra relación por origen geográfico, con la excepción de las accesiones provenientes de departamento del Quindío localizadas en la rama II. Las accesiones TolLig01, CalLi01 y AntLig01 conforman la rama I y se caracterizan por poseer frutos de buen tamaño, alto contenido de pulpa+semilla y bajo contenido de jugo. En la rama II se agrupa el mayor número de accesiones (25), con carac- terísticas promedio de calidad. La ramificación III está conformada por las accesiones TolLig02, CunLig03 y CunLig04, las cuales poseen frutos con cáscara gruesa, contenido de pulpa+semilla superior al $50 \%$ y alto contenido de ${ }^{\circ} \operatorname{Brix}(>15,8)$. En la última rama (IV), se concentra la mayor cantidad de accesiones con los mejores parámetros de calidad del fruto e incluyendo seis de las siete accesiones élite identificadas. Esta amplia distribución de los individuos de una misma accesión dentro y entre las ramas sugiere una relativa variabilidad pomológica que puede estar asociada con la naturaleza alogama de la granadilla y al intercambio de semillas entre diferen-

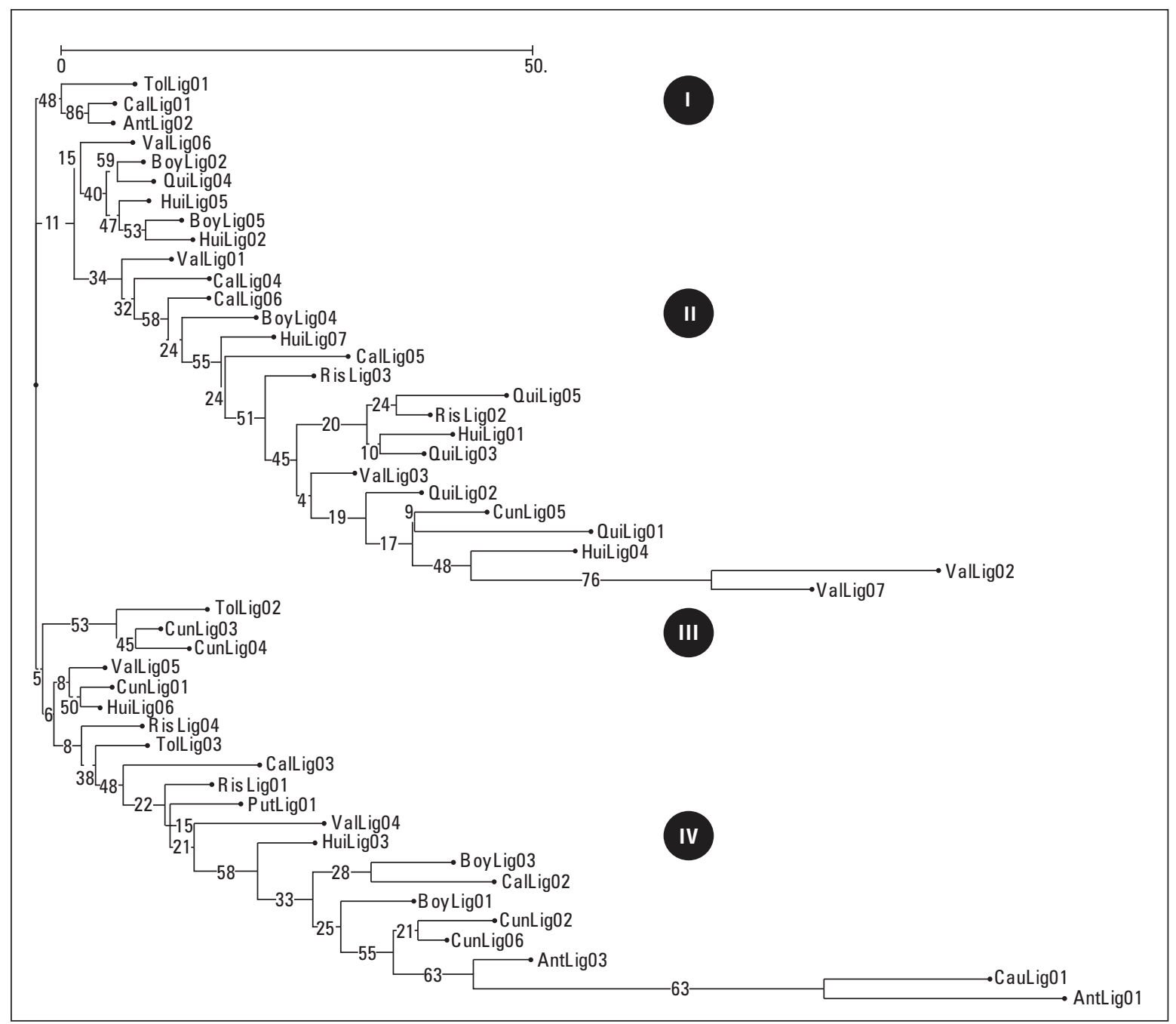

Figura 4. Clasificación arbórea (distancias euclidianas) de 48 accesiones de granadilla recolectadas a partir de algunos descriptores cuantitativos del fruto. Accesiones recolectadas: Ant (Antioquia), Boy (Boyacá), Cal (Caldas), Cau (Cauca), Cun (Cundinamarca), Hui (Huila), Put (Putumayo), Qui (Quindío), Ris (Risaralda), Tol (Tolima), Val (Valle del Cauca). 
tes departamentos. Además, la diferenciación de las accesiones élite podría ser aprovechada en procesos de hibridación con el propósito de concentrar genes favorables que puedan responder a problemas fitosanitarios y de adaptabilidad al cambio climático.

\section{DISCUSIÓN}

Las plantaciones de granadilla en Colombia son establecidas principalmente bajo el sistema de soporte en emparrado (96\%), donde la mayoría de agricultores reconocen las ventajas de este para la producción de frutos de buena calidad y la cosecha (Castro, 2001). Sin embargo, para Rivera et al. (2002) este sistema de soporte solo es apropiado para terrenos con pendiente inferior al $40 \%$, ya que facilita las labores del cultivo. Esta observación contrasta con las observaciones de campo en este estudio, donde la mayoría de los cultivos están establecidos en pendientes mayores al $60 \%$ y bajo el sistema de emparrado. Los beneficios del uso de la guadua para la construcción del sistema de conducción son reconocidos por la mayoría de los agricultores, debido a su bajo costo y alta durabilidad (Bernal y Tamayo, 1999). Un aspecto importante, fue el arreglo granadilla-café observado en algunas zonas, lo cual resalta la capacidad de los agricultores para proponer alternativas tecnológicas que permitan un mejor aprovechamiento del terreno. Este sistema ha mostrado claros indicadores sobre su eficiencia económica, social y ambiental, y a su vez ha permitido a las familias rurales afrontar los costos de producción y adquirir infraestructura para el beneficio del café (Nieto y Rivera, 2002). Por otro lado, las diferencias en las técnicas de siembra y manejo del cultivo son un reflejo de una domesticación incipiente en la granadilla. Lo anterior es soportado por la variabilidad en la germinación de la semilla (79,5 a 95,2\%) y el vigor de las plántulas de diferentes accesiones durante el mismo proceso (Posada et al., 2014). Otra característica relevante, es la au- sencia de la tecnología de riego en los cultivos visitados y la dependencia de la precipitación para satisfacer las necesidades hídricas. Según Ocampo et al. (2013), el sistema de riego por goteo en el cultivo de maracuyá ha presentado resultados favorables en el buen desarrollo de las plantaciones en épocas de sequía prolongada.

En las plantaciones de granadilla visitadas, antracnosis fue la enfermedad de mayor incidencia con un $23 \%$, siendo observada con mayor frecuencia en los departamentos de Boyacá, Caldas, Antioquia y Quindío, atacando hojas, tallos y frutos. Otra de la mayores limitantes del cultivo es el patógeno del suelo denominado secadera (Haematonectria haematococca) con un $17 \%$ de incidencia, la cual penetra por la raíces y causa una muerte ascendente en la planta. La distribución de esta enfermedad tuvo un mayor impacto en las zonas donde la granadilla se ha cultivado por más de 10 años y en plantaciones que son abonadas con gallinaza tratada inadecuadamente. De acuerdo con Fisher y Rezende (2008), la secadera es el principal problema fitosanitario en las especies cultivadas de Passiflora, ya que causa los mayores impactos en la calidad de la cosecha con pérdidas económicas.

En relación a los insectos plaga, la mosca del ovario fue la de mayor importancia con una incidencia del $34 \%$, causando caída de botones florales y frutos en los primeros estados de desarrollo (Hernández et al., 2011). Aunque esta plaga fue observada en los diferentes rangos de altitud, es más frecuente por debajo de los 2.000 msnm, y es observada en otras pasifloras cultivadas. Los trips son otra plaga de gran importancia económica en las especies frutales y en este estudio fue registrada en el 19\% de las fincas visitas, causando encrespamiento en los brotes terminales de las ramas y deteniendo el crecimiento (Rivera et al., 2002; Hernández et al., 2011). En el maracuyá, este insecto puede afectar hasta el $95 \%$ de los terminales vegetativos y el $75 \%$ de los botones florales, con pérdidas hasta $311,34 \mathrm{~kg} \mathrm{ha}^{-1}$ (Santos et al., 2011). 


\section{Estructura de la variabilidad genética}

Las encuestas con los productores mostraron que en Colombia existen cuatro regiones como centros principales de dispersión de semillas de granadilla: Urrao (Antioquia), Génova (Quindío), Buenavista (Boyacá) y Palestina (Huila). Estos centros están relacionados con las zonas de mayor producción y, por ende, son los proveedores a las demás regiones del país. Sin embargo, el municipio de Urrao fue donde se iniciaron los cultivos de granadilla en Colombia y en los años 80 y 90 abastecían el 90\% de la granadilla en el país (Bernal, 1991; Rivera et al., 2002). La disminución de los cultivos en esta zona del país fue causada por el ataque de la secadera y muchos de los productores migraron a otros departamentos (Caldas, Quindío y Risaralda), llevando el conocimiento y la tecnología. Lo anterior sugiere, que los productores de Urrao han contribuido considerablemente en los procesos de domesticación de la granadilla a través de una selección empírica de las plantas mejor adaptados a cada zona en particular.

En este estudio se han identificado siete accesiones élite con características sobresalientes de calidad (peso del fruto, ${ }^{\circ}$ Brix, porcentaje de pulpa+semilla y jugo) con una relativa variabilidad fenotípica. La calidad de la granadilla colombiana ha sido reconocida y países como Ecuador, Perú y México han introducido material proveniente del país, debido a que las selecciones locales presentan frutos de menor tamaño. En este sentido, Franco et al. (2008) en los estudios de caracterización de frutos provenientes de dos zonas productoras de México, mostraron una buen calidad interna, pero el peso no superó los $75 \mathrm{~g}$. En contraste, el peso promedio del fruto reportado en este estudio fue de $143 \mathrm{~g}$ y solamente la accesión ValLig07 (Valle del Cauca) colectada en estado silvestre registro un peso de 83,6 g.

Por otro lado, los resultados encontrados en este estudio son similares a los reportados por
Ocampo et al. (2013) en el maracuyá, donde existe una relativa variabilidad pomológica sin una estructuración de origen geográfica de las accesiones. Adicionalmente, los mismos autores mencionan que los parámetros de selección están asociados al peso y longitud del fruto, tal como se demuestra en este estudio y apoya lo reportado por Franco et al. (2008) en granadilla. En contraste, Medina et al. (2000) usando caracteres morfológicos reportan baja variabilidad en accesiones de granadilla y concluyen que puede ser debido a que las accesiones provienen de un área geográfica estrecha. Similarmente, Castañeda (2008), usando marcadores RAPD y AFLP, indica que la granadilla cultivada en los departamentos del Caldas, Quindío Risaralda presenta una estrecha variabilidad genética. Por otro lado, los resultados de esta investigación soportan los obtenidos por Bernal et al. (2014) con marcadores microsatélites y confirman que la granadilla cultivada en Colombia es fuente de viabilidad genética que puede ser aprovechada en programas de fitomejoramiento.

\section{Perspectivas para el fitomejoramiento}

Un programa de mejoramiento genético está basado en la búsqueda de variabilidad asociada a genes de interés dentro del acervo genético primario con el propósito de identificar genotipos superiores potencialmente útiles (Faleiro et al., 2005; Cerqueira-Silva et al., 2014). Es importante resaltar que Colombia forma parte del centro de origen (Killip, 1938), lo que brinda la oportunidad para tener una amplia variabilidad genética de la especie. Así mismo, es necesario explotar los recursos genéticos de sus parientes silvestres más cercanos como P. tiliifoliae L., P. magnifica L.K. Escobar, P. seemanni Griseb, $P$. fieldiana S. Tillet, $P$. palenquensis Holm-Niels. \& Lawesson, P. platyloba Killip y P. triloba Ruiz \& Pav. ex DC. con el propósito de aumentar la variabilidad de la especie con características agronómicas de interés que permitan afrontar problemas limitantes del cultivo (Coppens d'Eeckenbrugge, 2003; 
Yockteng et al., 2011). En este estudio se han colectado 48 accesiones de granadilla de diferentes orígenes geográficos, las cuales se diferencian por la forma redonda o alargada, el tamaño del fruto y variación en el color de la pulpa (figura 5). En este contexto, un programa de mejoramiento podría estar basado inicialmente en la selección de progenies por calidad del fruto con base en índices multivariados (Oliveira et al., 2008). Adicionalmente, se requiere la realización de estudios de caracterización agromorfológica y molecular (secuenciación) de este reservorio genético que diferencien o relacionen las accesiones evaluadas bajo un mismo ambiente y permitan establecer el grado variabilidad genética total.

\section{CONCLUSIONES}

En las colectas del material vegetal en las fincas de productores se identificaron seis insectos plaga e igual número de enfermedades que afectan el desarrollo de las plantaciones. Sin embargo, la mosca del ovario (Dasiops spp.) y la roña (Colletotrichum gloeosporioides) y la secadera (Haematonectria haematococca) fueron las de mayor incidencia e impacto en los cultivos de granadilla.

Las encuestas a los productores permitieron establecer cuatro centros geográficos principales de dispersión de semillas en diferentes zonas del país. Sin embargo, la de mayor representatividad

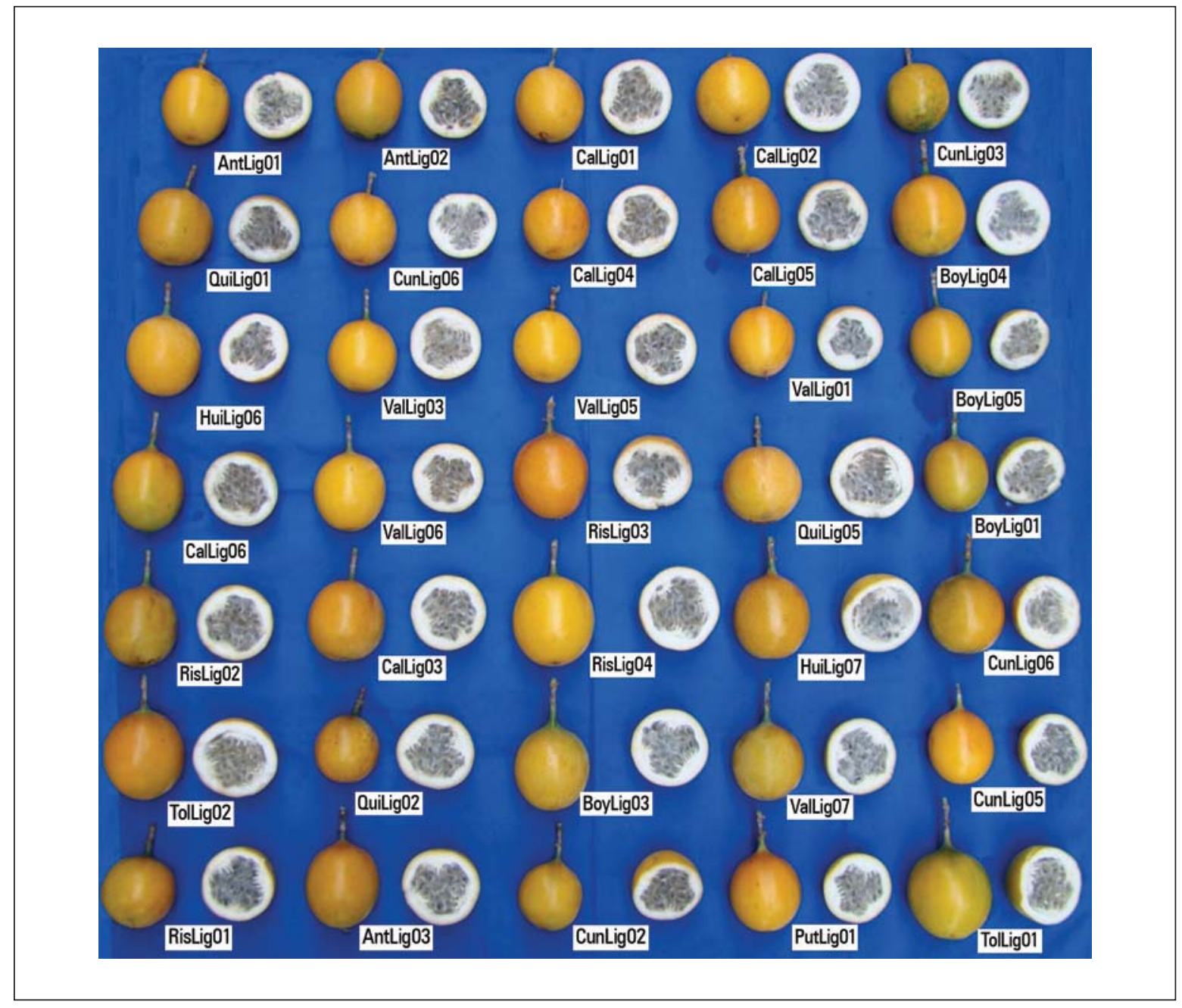

Figura 4. Diversidad en forma, tamaño y color de las accesiones colectadas de granadilla (P. ligularis) en Colombia. Foto: J. Ocampo. 
está localizada en el municipio de Urrao (Antioquia), debido a que la granadilla se cultiva por más de 50 años en esta región.

La caracterización de los frutos colectados permitió identificar seis accesiones élite con alta calidad proveniente de cinco departamentos con alta calidad para la comercialización. Sin embargo, tres de ellas provienen del departamento de Cundinamarca (CunLig02, CunLig03 y CunLig06), lo que sugiere que los cultivos de esta zona son promisorios para la selección varietal.

El análisis de clasificación mostró que existe una relativa variabilidad intraespecífica en la forma y tamaño de los frutos en las 48 accesiones colectas. Sin embargo, esta variabilidad no está estructurada de acuerdo con el origen geográfico, lo que puede ser consecuencia del fenómeno de alogamía y al intercambio de semillas entre productores de diferentes regiones.

El conjunto de resultados sugieren que los genotipos seleccionados pueden ser usados en pro- gramas de mejoramiento genético en búsqueda de cultivares de mayor producción y tolerancia a problemas fitosanitarios. Sin embargo, deben ser evaluados y caracterizados agronómicamente para establecer la variabilidad total presente en la granadilla.

\section{AGRADECIMIENTOS}

Esta investigación fue posible por el financiamiento del Ministerio de Agricultura y Desarrollo Rural de Colombia (MADR, Bogotá) bajo el marco del Proyecto Aprovechamiento de la Diversidad del Maracuyá, la Gulupa y la Granadilla para mejorar y diversificar los sistemas de producción en Colombia 0742008L6772-3447. Los autores agradecen a los productores y asistentes técnicos de los Centros Provinciales (CPGA) y Umatas y por su colaboración en los trabajos de campo. Adicionalmente, a la estudiante de la Universidad de Caldas, Nathali López, por su apoyo en el procesamiento de datos.

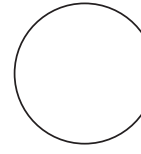

Agronet. 2014. Ministerio de Agricultura y Desarrollo Rural de Colombia, Análisis - Estadísticas, Granadilla. En: http://www.agronet.gov.co, consulta: octubre de 2014.

Arias, J.C., J. Ocampo y R. Urrea. 2015. Sistemas de polinización en la Granadilla (Passiflora ligularis Juss.) como base para estudios genéticos y de conservación. Acta Agron. (en imprenta).

Bernal, J.A y P.J. Tamayo, 1999. Informe de visita a municipios productores de granadilla del departamento de Caldas. Corpoica Regional 4, Rionegro, Colombia.

Bernal, J.A. 1991. Biología floral de dos especies de pasifloras. pp. 153-164. En: Libro de resumenes, Primer Simposio Internacional de Passifloras. Universidad Nacional de Colombia. Palmira, Colombia.

Bernal, N., J. Ocampo y J. Hernández. 2014. Caracterización y análisis de la variabilidad genética de la granadilla (Passiflora ligularis Juss.) en Colombia empleando marcadores microsatélites. Rev. Bras.

\section{REFERENCIAS BIBLIOGRÁFICAS}

Frutic. 36(3), 598-611. Doi: 10.1590/0100-2945$251 / 13$

Castañeda, C.A. 2008. Evaluación de la diversidad genética de materiales cultivados de Passiflora liguraris procedentes del eje cafetero. Trabajo de grado. Facultad de Ciencias, Pontificia Universidad Javeriana, Bogotá.

Castro, J.J. 2001. Guía básica para el establecimiento y mantenimiento del cultivo de la granadilla (Passiflora ligularis Juss.). Asohofrucol, Fondo Nacional del Fomento Hortofrutícola, Bogotá.

Cerqueira-Silva, C.B., O.N. Jesus, E.S. Santos, R.X. Corrêa y A.P. Souza. 2014. Genetic breeding and diversity of the genus Passiflora: Progress and perspectives in molecular and genetic studies. Int. J. Mol. Sci. 15, 14122-14152. Doi:10.3390/ijms150814122

Coppens d'Eeckenbrugge, G. 2003. Exploração da diversidade genética das passifloras. pp. 24-27. En: Libro de Resúmenes. Sexto Simpósio Brasileiro sobre a 
Cultura do Maracujazeiro. Campos de Goytacazes, Brasil.

Faleiro, F. G., N.T. Junqueira, M.F. Braga y J.R. Peixoto. 2005. Germoplasma e melhoramento genético do maracujazeiro - Desafíos da pesquisa. pp. 185-209. En: Faleiro, F.G., N.T Junqueira y B.F. Braga (eds.). Maracujá: germoplasma e melhoramento genético. Embrapa Cerrados, Planaltina, DF, Brasil.

Fischer, I.H y J.A. Rezende. 2008. Diseases of passion flower (Passiflora spp.). Pest Techn. 2(1), 1-19.

Flórez, L.M., L.V. Pérez, L.M. Melgarejo y S. Hernández. 2012. Caracterización fisicoquímica, fisiológica y bioquímica del fruto de gulupa (Passiflora edulis Sims) como indicadores para el punto óptimo de cosecha (pp. 53-79). En: Melgarejo, L.M (ed.). Ecofisiología del cultivo de la gulupa (Passiflora edulis Sims). Universidad Nacional de Colombia, Bogotá.

Franco, O., J.R. Tobar, R. Quijano y A. González. 2008. Caracterización preliminar de frutos de granada china (Passiflora ligularis Juss.) en Hueyapan y Teziutlán, Puebla. Ciencia Ergo Sum 15(1), 54-60.

Franco, Y., F. Alzate y J.M. Peláez. 2007. Factores ambientales incidentes en la población de Xylocopa y su efecto en el cultivo de granadilla en tres veredas del municipio de Guarne (Colombia). Rev. Uni. Cat. Ori. 24, 73-88.

Hammer, K., F. Heuser, K. Khoshbakht y Y. Teklu. 2012. Plant genetic resources for plant breeding. pp. 87-107. En: Acquaah, G. (ed.). Principles of plant genetics and breeding. $2^{\text {nd }}$ ed. Wiley/Blackwell, Chichester, UK.

Hernández, L. M., C.F. Castillo, J. Ocampo y K. Wyckhuys. 2011. Guía de identificación de las principales plagas y enfermedades del maracuyá, la gulupa y la granadilla. Centro Bio-Sistemas Universidad Jorge Tadeo Lozano, Bogotá; Centro Internacional de Agricultura Tropical (CIAT), Cali, Colombia.

Hidalgo, R. 2003. Variabilidad genética y caracterización de especies vegetales. pp. 2-26. En: Franco, T.L y R. Hidalgo (eds.). Análisis estadístico de datos de caracterización morfológica de recursos fitogenéticos. Boletín Técnico No. 8. Instituto Internacional de Recursos Fitogenéticos (IPGRI), Cali, Colombia.

Hill, T. y P. Lewicki. 2007. Statistics: Methods and applications. StatSoft, Tulsa, OK.

Icontec. 1997. Norma 4101. Frutas frescas. Granadilla. Especificaciones. Instituto Colombiano de Normas Técnicas y Certificación, Bogotá.

Killip, E.P. 1938. The American species of Passifloraceae. Bot. Ser. 19 (1, 2), 1-163.
Medina, C.I., M. Lobo y R. Correa. 2000. Caracterización morfológica y química de Pasifloras Andinas como apoyo al desarrollo de estas especies. pp. 1318. En: Memorias III Seminario de Frutas de Clima Frío Moderado. Corpoica, Manizales, Colombia.

Nieto, A.M y B. Rivera. 2002. El arreglo café-granadilla: una alternativa tecnológica para la innovación de los sistemas productivos. pp. 230-237. En: Libro de resúmenes. IV Seminario de Frutas de Clima Frío Moderado. Corpoica. Medellín, Colombia.

Ocampo, J., G. Coppens d'Eeckenbrugge, C. Olano y R. Schnell. 2004. AFLP analysis for the study of genetic relationships among cultivated Passiflora species of the subgenera Passiflora and Tacsonia. Proc. Interam. Soc. Trop. Hortic. 47, 72-76.

Ocampo, J. 2007. Study of the genetic diversity of genus Passiflora L. and its distribution in Colombia. Tesis de doctorado. Centre International d'Etudes Supérieures en Sciences Agronomiques (SupAgro), Montpellier, Francia.

Ocampo, J. y K. Wyckuys. 2010. Mejoramiento genético participativo de la granadilla (Passiflora ligularis Juss.) en Colombia. p. 127. En: Memorias Primer Congreso Latinoamericano de Passiflora. Corporación Centro de Desarrollo Tecnológico de las Pasifloras de Colombia (Cepass), Neiva, Colombia.

Ocampo, J., R. Urrea, K. Wyckuys y M. Salazar. 2013. Exploración de la variabilidad genética del maracuyá (Passiflora edulis f. flavicarpa Degener) como base para un programa de mejoramiento en Colombia. Acta Agron. 62(4), 352-360.

Oliveira, E. J., V. Santos, D.S. Lima, M.D. Machado, R.S. Lucena, T.B.N. Motta y M. Castellen. 2008. Selecao em progenies de maracujazeiro-amarelo com base em índices multivariados. Pesq. Agropec. Bras. 43(11), 1543-1549. Doi: 10.1590/S0100204X2008001100013

Parra, M. 2013. Acuerdo de competitividad para la cadena productiva de pasifloras en Colombia. Asohofrucol, Cepass, Consejo Nacional de Pasifloras, Ministerio de Agricultura y Desarrollo Rural, Bogotá.

Parra, M., A. Aguilera, W. Escobar, V. Rubiano y A. Rodríguez. 2011. Agenda prospectiva de investigación y desarrollo tecnológico para la cadena productiva de granadilla en el departamento del Huila. Corporación Cepass, Neiva; Universidad del Valle, Cali; Ministerio de Agricultura y Desarrollo Rural, Bogotá.

Perrier, X. y J.P. Jacquemoud-Collet. 2006. DARwin software. En: http://darwin.cirad.fr; consulta: septiembre de 2014 
Posada, P., J. Ocampo y L.G. Santos. 2014. Estudio del comportamiento fisiológico de la semilla de tres especies cultivadas de Passiflora L. (Passifloraceae) como una contribución para la conservación ex situ. Rev. Colomb. Cienc. Hortic. 8(1), 9-19. Doi: 10.17584/rcch.2014v8i1.2796

Rivera, B., D. Miranda, L.A. Ávila y A.M. Nieto. 2002. Manejo integral del cultivo de la granadilla (Passiflora ligularis Juss). Ed. Litoas, Manizales, Colombia.

Santos, O., E.H. Varón y A. Floriano. 2011. Nivel de daño económico y umbral de acción para Neohydatothrips signifer en maracuyá (Passiflora edulis Degener) var. flavicarpa en el departamento del Huila, Colombia. pp. 83-98. En: Varón, E.H., M. Buenaventura y O. Santos (eds.). Manual técnico en maracuyá. Corpoica, C.I. Nataima, El Espinal, Colombia.

Santos, L.F., E.J. Oliveira, A. Santos, F.M. Carvalho, J.L. Costa y J.G. Pádua. 2011. ISSR markers as a tool for the assessment of genetic diversity in Passiflora. Biochem. Gen. 49(7-8), 540-54. Doi: 10.1007/ s10528-011-9429-5

Segura, S., G. Coppens d'Eeckenbrugge, A. Bohórquez, P. Ollitrault y J. Tohmé. 2002. An AFLP study of the genus Passiflora focusing on subgenus Tacsonia. Gen. Resour. Crop Evol. 49, 111-123. Doi: 10.1023/A:1014731922490

Snow, N. y J. MacDougal, J. 1993. New chromosome reports in Passiflora (Passifloraceae). Syst. Botany 18(2), 261-273. Doi: 10.2307/2419402

Tamayo, P.J. y F. Varón. 1996. Manejo de problemas patológicos en los cultivos de frijol y granadilla en el municipio de Urrao, Antioquia. pp. 11-21. En: Manejo de algunos problemas fitosanitarios en Arveja, Frijol, Granadilla, Repollo, Coliflor y Brócoli. Boletín de Sanidad Vegetal. ICA, Bogotá.

Yockteng, R., G. Coppens d'Eeckenbrugge y T. SouzaChies. 2011. Passiflora L. pp. 129-171. En: Chittaranjan, K. (ed.). Wild crop relatives: Genomic and breeding resources. tropical and subtropical fruits. Springer Verlag, Berlin

Yotoko, S.C., M.C. Dornelas, P.D.Togni, T.C. Fonseca, F.M. Salzano, S.L. Bonatto y L.B. Freitas. 2011 Does variation in genome sizes reflect adaptive or neutral processes? New clues from Passiflora. PloS One 6(3), e18212. Doi: 10.1371/journal. pone.0018212 